\title{
On the Nonlinear Distortions of Sound and its Coupling with Other Modes in a Gaseous Plasma with Finite Electric Conductivity in a Magnetic Field
}

\author{
Anna PERELOMOVA \\ Faculty of Applied Physics and Mathematics \\ Gdańsk University of Technology \\ Narutowicza 11/12, 80-233 Gdańsk, Poland; e-mail: anpe@mif.pg.gda.pl
}

(received November 28, 2015; accepted July 10, 2016)

\begin{abstract}
Nonlinear phenomena of the planar and quasi-planar magnetoacoustic waves are considered. We focus on deriving of equations which govern nonlinear excitation of the non-wave motions by the intense sound in initially static gaseous plasma. The plasma is treated as an ideal gas with finite electrical conductivity permeated by a magnetic field orthogonal to the trajectories of gas particles. This introduces dispersion of a flow. Magnetoacoustic heating and streaming in the field of periodic and aperiodic magnetoacoustic perturbations are discussed, as well as generation of the magnetic perturbations by sound. Two cases, corresponding to magnetosound perturbations of low and high frequencies, are considered in detail.
\end{abstract}

Keywords: magnetoacoustics; nonlinear wave phenomena; finite electrical conductivity; acoustic dispersion; acoustic heating and streaming.

\section{Introduction}

Magnetohydrodynamic phenomena in conducting fluids attract attention of researchers in cosmic physics, geophysics, plasma physics, physics of controlled thermonuclear fusion, and hypersonic aerodynamics. Magnetic strength, which is not strictly parallel to the fluid velocity, enlarges the fluid's stiffness; this in turn enlarges the speed of sound (HERLOFson, 1950; TRuesdell, 1950). Acoustic anisotropy of magnetic media is the most important issue which essentially complicates the mathematical context of magnetohydrodynamics (MHD). It was established in 50-ties of the last century that finite conductivity of plasma introduces dispersion and absorption (dependent on frequency) of sound planar waves whose propagation direction is perpendicular to the direction of the magnetic field (ANDERSON, 1953). This is the physical dispersion which appears due to magnetic effects. We do not consider in this study geometrical dispersion which follows propagation of waves in bounded volumes and waveguides, and which in fact introduces the characteristic length scale in addition to the wavelength of perturbations (LEBLE, 1991). An unbounded volume of gas is considered. We do not consider dispersion caused by external forces or specific heating/cooling which make the background of wave propagation non-uniform either. These effects are well-studied in the context of MHD and have been discussed in many papers, see for example (ANAND, YADAV, 2014; Vincenti, BALDWIN JR, 1962; Ponomarev, 1961; Fabian et al., 2005).

Magnetoacoustic travelling waves transport energy and momentum. They can heat or accelerate the plasma. Making use of that, we may conclude about properties of plasma observing magnetoacoustic heating and streaming. The experimental data may serve as a unique tool for plasma diagnostics. The obligatory conditions for transporting energy and momentum from wave motion into the entropy and vortex modes are both nonlinearity and attenuation. In this study, we account for attenuation and dispersion which originate exclusively from the finite electrical conductivity of a plasma. Newtonian attenuation due to shear and bulk viscosity, as well as thermal conductivity or any internal relaxation processes in a gas, are left out of account.

The primary intention is to describe the nonlinear distortions of the magnetoacoustic wave itself. The nonlinear distortion of planar sound in which gas particles move perpendicularly to magnetic field are wellestablished in the case of perfectly conducting gas, involving waveforms with discontinuities (SINGH et al., 2011; 2012; Sharma et al., 1987; Geffen, 1963). A short review of the problems relating to wave propa- 
gation with consideration of nonlinear phenomena may be found in the study (SHYAM et al., 1981). As for gases with finite electrical conductivity, there is evident lack in the nonlinear description, though the linear dispersion relation, which in fact determines linear dynamics of magnetoacoustic perturbations, has been established long ago by ANDERSON (1953). This lack may be probably explained by frequency-dependent behaviour which makes non-linear analysis without additional simplifications difficult (KRISHNA PASAD et al., 2014) and many other conditions of fluid flows in a plasma which have come into notice of researchers. ANDERSON (1953) considers weak magnetic strength and linear evolution, which in fact makes the results limited and valid in the leading order.

Once nonlinear dynamics of magnetosound wave is established, its nonlinear losses in energy and momentum may be considered. The nonlinear interaction of magnetohydrodynamic waves has been the subject of interest of numerous authors (SAGDEEV, GALEEV, 1969; Petviashvili, Pokhotelov, 1992; Shukla, Stenflo, 1999; Brodin et al., 2006). We may mention PonOMAREv (1961) who has attracted attention to nonlinear transfer of energy between magnetohydrodynamic waves and other types of waves in plasma with finite conductivity (PonOMAREv, 1961). Amplification of Alfvén wave due to nonlinear interaction with a magnetoacoustic wave has been discovered recently in (ZAVERshinsky, Molevich, 2014). Usually, an analysis concerns three-wave interactions and depends on spectra of interacting waves. The review by BALLAI (2006) summarises knowledge on nonlinear waves in solar plasmas. It also considers nonlinear resonant waves.

In contrast, the method which has been applied by the author in a number of hydrodynamic problems concerning flows of fluids with various attenuation, does not sort with spectra of interacting waves but allows to derive a set of dynamic equations which are valid for any kind of interacting modes, see, for example, (Perelomova, 2006). The description of nonlinear interaction of different types of magnetohydrodynamic motions imposes resolution of some issues:

1) to determine the MHD modes in a flow of infinitely-small perturbations, as linear links between specific perturbations;

2) to derive the leading-order nonlinear equations which describe coupling of modes in weakly nonlinear flow;

3) to solve them relating to the physical context of a problem.

The main idea is to establish linear projectors which distinguish in the total perturbations only one specific mode. They also eliminate all other modes in the linear parts of dynamic equations while applying on the system of conservation equations in the differential form. On the whole, the procedure appoints a sequence of actions to obtain results as a series in powers of the Mach number $M$ with any desired accuracy. All evaluations in this study are made within accuracy up to quadratic nonlinear terms, that is, up to terms proportional to $M^{2}$, which are of the major importance in weakly nonlinear fluid flows. The corrected nonlinear links recalling that in the Riemann wave will be established, and equations which describe generation of the secondary modes in the dominative magnetoacoustic field, will be derived and discussed.

\section{Magnetoacoustic and non-wave modes in a planar flow of infinitely-small magnitude}

\subsection{PDEs describing planar flow of a conducting gas}

We consider a planar flow of a gas whose velocity $v(x, t)$ is perpendicular to the magnetic field strength $\mathbf{H}=(0,0, H(x, t))$, where $t$ and $x$ denote time and Carthesian coordinate indicating the axis orthogonal to the magnetic field, respectively. The magnetic field is evidently solenoidal, $\boldsymbol{\nabla} \cdot \mathbf{H}=0$. The hydrodynamic flow equations (KorobeINIKOV, 1976) will be the starting point:

$$
\frac{\partial \rho}{\partial t}+\frac{\partial \rho v}{\partial x}=0
$$

for mass,

$$
\rho\left(\frac{\partial v}{\partial t}+v \frac{\partial v}{\partial x}\right)+\frac{\partial p}{\partial x}+\frac{\partial h}{\partial x}=0
$$

for momentum,

$$
\frac{\partial s}{\partial t}+v \frac{\partial s}{\partial x}=0
$$

for entropy $s$, and

$$
\frac{\partial h}{\partial t}+v \frac{\partial h}{\partial x}+2 h \frac{\partial v}{\partial x}+\beta\left(-\frac{\partial^{2} h}{\partial x^{2}}+\frac{1}{2 h}\left(\frac{\partial h}{\partial x}\right)^{2}\right)=0
$$

for the magnetic pressure $h$, where

$$
h=\mu H^{2} / 2
$$

$\rho, p$ are density and pressure of a gas, respectively. In Eq. (4), $\beta=(\mu \sigma)^{-1}, \mu$ is the magnetic permeability, and $\sigma$ is the electrical conductivity of a fluid. Equation (4) readily follows from the equation

$$
\frac{\partial \mathbf{H}}{\partial t}-\nabla \times(\mathbf{v} \times \mathbf{H})=\beta \Delta \mathbf{H} .
$$

\subsection{Projecting of the total perturbation into specific modes}

Equations (1)-(4) should be completed by the caloric equation of state and the thermodynamic identity for equilibrium thermodynamic processes, 
$T \mathrm{~d} s=\mathrm{d} e+p \mathrm{~d}\left(\rho^{-1}\right)$ ( $T$ denotes temperature $)$. We make use of the internal energy $e$ of an ideal gas:

$$
e=\frac{p}{(\gamma-1) \rho}
$$

where $\gamma$ is the ratio of specific heats under constant pressure and constant density. The unperturbed quantities will be marked by subscript 0 , and all disturbances will be primed. Perturbations are developed against the motionless background with $v_{0}=0$. In terms of velocity and perturbations in density, pressure, and magnetic pressure, Eqs. (1)-(4) take the leading-order form:

$$
\frac{\partial \psi}{\partial t}+L \psi=\psi_{n l}
$$

where

$$
\begin{aligned}
& \psi=\left(\begin{array}{c}
\rho^{\prime} \\
v \\
p^{\prime} \\
h^{\prime}
\end{array}\right), \\
& L=\left(\begin{array}{cccc}
0 & \rho_{0} \frac{\partial}{\partial x} & 0 & 0 \\
0 & 0 & \frac{1}{\rho_{0}} \frac{\partial}{\partial x} & \frac{1}{\rho_{0}} \frac{\partial}{\partial x} \\
c_{0}^{2} \rho_{0} \frac{\partial}{\partial x} & 0 & 0 & 0 \\
2 h_{0} \frac{\partial}{\partial x} & 0 & 0 & -\beta \frac{\partial^{2}}{\partial x^{2}}
\end{array}\right), \\
& \psi_{n l}=\left(\begin{array}{c}
-\rho^{\prime} \frac{\partial v}{\partial x}-v \frac{\partial \rho^{\prime}}{\partial x} \\
-v \frac{\partial v}{\partial x}+\frac{\rho^{\prime}}{\rho_{0}^{2}} \frac{\partial p^{\prime}}{\partial x}+\frac{\rho^{\prime}}{\rho_{0}^{2}} \frac{\partial h^{\prime}}{\partial x} \\
-v \frac{\partial p^{\prime}}{\partial x}-\gamma p^{\prime} \frac{\partial v}{\partial x} \\
-v \frac{\partial h^{\prime}}{\partial x}-2 h^{\prime} \frac{\partial v}{\partial x}-\frac{\beta}{2 h_{0}}\left(\frac{\partial h^{\prime}}{\partial x}\right)^{2}
\end{array}\right)
\end{aligned}
$$

and $c_{0}$ denotes the infinitely small signal sound speed $c$ in an ideal gas at unperturbed thermodynamic state $p_{0}, \rho_{0}$ in the absence of magnetic field,

$$
c=\sqrt{\frac{\gamma p}{\rho}} .
$$

The linear system

$$
\frac{\partial \psi}{\partial t}+L \psi=0
$$

determines four eigenvectors. Two of them correspond to the magnetoacoustic modes which propagate in the positive direction of axis $x$ (first fast magnetosound wave) or in the negative direction of axis $x$ (second fast magnetosonic mode): the entropy mode (third) and the Alfvén wave, which is stationary in the case of perpendicular to velocity magnetic field (ordered as fourth). The analysis of Eq. (5) in the context of nonlinear effects of sound depends on frequency of the dominativing sound.

\subsection{Low frequencies}

This case concerns nonlinear effects of sound with

$$
\beta \omega \frac{c_{m, 0}^{2}-c_{0}^{2}}{c_{m, 0}^{4}} \ll 1
$$

where

$$
c_{m}=\sqrt{c^{2}+c_{A}^{2}}, \quad c_{A}=\sqrt{2 h / \rho}
$$

denote the magnetosonic speed and the Alfvén speed, respectively, and $c_{m, 0}$ denotes $c_{m}$ at the unperturbed state $p_{0}, \rho_{0}$. No restrictions concerning the smallness of unperturbed magnetic pressure $h_{0}$, and, hence, $c_{m, 0}^{2} / c_{0}^{2}-1$ have been set. Considering any perturbation as a planar wave proportional to $\exp (i \omega t-i k x)$, one arrives at dispersion relations

$$
\begin{aligned}
& \omega_{1}=c_{m, 0} k+\frac{i \beta\left(c_{m, 0}^{2}-c_{0}^{2}\right)}{2 c_{m, 0}^{2}} k^{2}, \\
& \omega_{2}=-c_{m, 0} k+\frac{i \beta\left(c_{m, 0}^{2}-c_{0}^{2}\right)}{2 c_{m, 0}^{2}} k^{2}, \\
& \omega_{3}=0, \\
& \omega_{4}=\frac{i \beta k^{2} c_{0}^{2}}{c_{m, 0}^{2}} .
\end{aligned}
$$

The total perturbation is actually a sum of specific disturbances which represent the eigenvectors correspondent to eigenvalues $-i \omega_{n}(n=1, \ldots, 4)$ :

$$
\begin{aligned}
v= & \sum_{n=1}^{4} v_{n}=\frac{c_{m, 0}}{\rho_{0}} \rho_{1}^{\prime}-\frac{\beta\left(c_{m, 0}^{2}-c_{0}^{2}\right)}{2 c_{m, 0}^{2} \rho_{0}} \frac{\partial \rho_{1}^{\prime}}{\partial x} \\
& -\frac{c_{m, 0}}{\rho_{0}} \rho_{2}^{\prime}-\frac{\beta\left(c_{m, 0}^{2}-c_{0}^{2}\right)}{2 c_{m, 0}^{2} \rho_{0}} \frac{\partial \rho_{2}^{\prime}}{\partial x}-\frac{\beta c_{0}^{2}}{c_{m, 0}^{2} \rho_{0}} \frac{\partial \rho_{4}^{\prime}}{\partial x},
\end{aligned}
$$

$p^{\prime}=\sum_{n=1}^{4} p_{n}^{\prime}=c_{0}^{2} \rho_{1}^{\prime}+c_{0}^{2} \rho_{2}^{\prime}+c_{0}^{2} \rho_{4}^{\prime}$,

$$
\begin{aligned}
h^{\prime}= & \sum_{n=1}^{4} h_{n}^{\prime}=\frac{2 h_{0}}{\rho_{0}} \rho_{1}^{\prime}-\frac{\beta\left(c_{m, 0}^{2}-c_{0}^{2}\right)}{c_{m, 0}} \frac{\partial \rho_{1}^{\prime}}{\partial x} \\
& +\frac{2 h_{0}}{\rho_{0}} \rho_{2}^{\prime}+\frac{\beta\left(c_{m, 0}^{2}-c_{0}^{2}\right)}{c_{m, 0}} \frac{\partial \rho_{2}^{\prime}}{\partial x}-c_{0}^{2} \rho_{4}^{\prime} .
\end{aligned}
$$


Index in summation $n$ denotes ordering number of the specific mode. The entropy mode is isobaric. Equations (10) are evaluated with accuracy up to terms proportional to $\beta^{0}$ and $\beta^{1}$ inclusively. Hence, we consider weak dispersion of the flow, and correspondent weak attenuation following dispersion. The rows which distinguish excess densities specifying the third and fourth roots,

$$
\begin{aligned}
& P_{3}\left(\rho^{\prime} \quad v \quad p^{\prime} \quad h^{\prime}\right)^{\mathrm{T}}=\rho_{3}^{\prime}, \\
& P_{4}\left(\rho^{\prime} \quad v \quad p^{\prime} \quad h^{\prime}\right)^{\mathrm{T}}=\rho_{4}^{\prime},
\end{aligned}
$$

may be readily established:

$$
\begin{aligned}
& P_{3}=\left(\begin{array}{llll}
1 & 0 & -\frac{1}{c_{0}^{2}} & 0
\end{array}\right), \\
& P_{4}=\left(\begin{array}{ll}
0 & \left.-\frac{\left(c_{m, 0}^{2}-c_{0}^{2}\right) \rho_{0} \beta}{c_{m, 0}^{4}} \frac{\partial}{\partial x} \frac{1}{c_{0}^{2}}-\frac{1}{c_{m, 0}^{2}}-\frac{1}{c_{m, 0}^{2}}\right) .
\end{array} .\right.
\end{aligned}
$$

They are also evaluated with accuracy up to terms proportional to $\beta^{0}, \beta^{1}$. $P_{3}, P_{4}$ reduce all terms of the other modes when applying in system (5). They yield the linear dynamic equations for $\rho_{3}^{\prime}$ and $\rho_{4}^{\prime}$, respectively.

\subsection{High frequencies}

Another limiting case is represented by inequality

$$
\beta \omega \equiv B^{-1} \omega \gg c_{m, 0}^{2}-c_{0}^{2} .
$$

We will conditionally call this limit "high-frequency": this case concerns also weak magnetic strengths, $c_{m, 0}^{2} / c_{0}^{2}-1 \ll 1$. Only terms proportional to zero and first powers of this small parameter, will be kept. All evaluations are undertaken with accuracy up to zero and first powers of $B, B^{0}$, and $B^{1}$. Recalling all steps described in the subsection before, one arrives at the dispersion relations

$$
\begin{aligned}
& \omega_{1}=c_{0} k+i \frac{B}{2}\left(c_{m, 0}^{2}-c_{0}^{2}\right), \\
& \omega_{2}=-c_{0} k+i \frac{B}{2}\left(c_{m, 0}^{2}-c_{0}^{2}\right), \\
& \omega_{3}=0 \\
& \omega_{4}=\frac{i k^{2}}{B}-i B\left(c_{m, 0}^{2}-c_{0}^{2}\right) .
\end{aligned}
$$

The total perturbation represents a sum of specific perturbations:

$$
\begin{aligned}
v= & \sum_{n=1}^{4} v_{n}=\frac{c_{0}}{\rho_{0}} \rho_{1}^{\prime}+\frac{B\left(c_{m, 0}^{2}-c_{0}^{2}\right)}{2 \rho_{0}} \int \rho_{1}^{\prime} \mathrm{d} x \\
& -\frac{c_{0}}{\rho_{0}} \rho_{2}^{\prime}+\frac{B\left(c_{m, 0}^{2}-c_{0}^{2}\right)}{2 \rho_{0}} \int \rho_{2}^{\prime} \mathrm{d} x-\frac{B}{\rho_{0}} \int h_{4}^{\prime} \mathrm{d} x \\
p^{\prime}= & \sum_{n=1}^{4} p_{n}^{\prime}=c_{0}^{2} \rho_{1}^{\prime}+c_{0}^{2} \rho_{2}^{\prime}, \\
h^{\prime}= & \sum_{n=1}^{4} h_{n}^{\prime}=B c_{0}\left(c_{m, 0}^{2}-c_{0}^{2}\right) \int \rho_{1}^{\prime} \mathrm{d} x \\
& -B c_{0}\left(c_{m, 0}^{2}-c_{0}^{2}\right) \int \rho_{2}^{\prime} \mathrm{d} x+h_{4}^{\prime} .
\end{aligned}
$$

The limits of integration should be chosen in accordance with the physical context of a flow. As usual, the magnetoacoustic perturbations for the first mode vanish at plus infinity, so the lower limit of integration equals $\infty$, and the upper one equals $x$. The projector $P_{3}$ is the same as in the low-frequency case, and the projector which distinguishes the magnetic pressure in the fourth mode, takes the form

$$
P_{4, h}=\left(\begin{array}{llll}
0 & -B\left(c_{m, 0}^{2}-c_{0}^{2}\right) \rho_{0} \int \mathrm{d} x & 0 & 1
\end{array}\right) .
$$

We will consider the non-linear generation of two non-wave modes by the dominative planar and quasiplanar sound in Secs. 4, 5. The main idea is to make use of linear definition of specific modes and to apply projecting in order to eliminate all other terms in the linear parts of dynamic equations.

\section{Nonlinear corrections in magnetoacoustic perturbations and dynamic equations}

Projection gives a possibility to derive weakly nonlinear equations because it distributes nonlinear terms in $\psi_{n l}$ between dynamic equations in a proper way. Making use of the physical conditions of a flow, the nonlinear terms which include cross contributions of all modes, may be selected. Among the most meaningful problems of nonlinear interactions sound is dominative, and only acoustic nonlinear terms may be considered among all variety of nonlinear terms. For definiteness, the first magnetoacoustic mode will be treated as dominative. This means that magnetoacoustic perturbations are much larger than that of the non-wave modes over spatial and temporal domains which are considered. This in fact determines spacial and temporal domains where results are valid. The nonlinear terms in dynamic equations for the nonwave modes form some kind of "acoustic forces"; they originate from loss in acoustic momentum and energy and may be readily established making use of 
projecting. The Riemann wave is known to be the waveform which is an exact solution of the conservation equations in flow of an ideal gas. The correspondent nonlinear links in the dominative magnetoacoustic wave should be established; they are necessary for correct distribution of the nonlinear terms between dynamic equations in the leading order by use of projecting.

\subsection{Low frequencies}

The linear eigenvector for the progressive in the positive direction of axis $x$ low-frequency magnetoacoustic modes (when $\beta$ tends to zero) takes the form:

$$
\begin{aligned}
\psi_{1} & =\left(\begin{array}{llll}
\rho_{1}^{\prime} & v_{1} & p_{1}^{\prime} & h_{1}^{\prime}
\end{array}\right)^{\mathrm{T}} \\
& =\left(\begin{array}{llll}
\frac{\rho_{0}}{c_{m, 0}} & 1 & \frac{\rho_{0} c_{0}^{2}}{c_{m, 0}} & \frac{\rho_{0}\left(c_{m, 0}^{2}-c_{0}^{2}\right)}{c_{m, 0}}
\end{array}\right)^{\mathrm{T}} v_{1} .
\end{aligned}
$$

The vector with unknown constants $K, L, N$,

$$
\psi_{1, n}=\left(\begin{array}{llll}
K_{m} & 0 & L_{m} & N_{m}
\end{array}\right)^{\mathrm{T}} v_{1}^{2},
$$

corrects $\psi_{1}$. The sum $\psi_{1}+\psi_{1, n}$ should result in four equivalent leading-order nonlinear equations for magnetoacoustic velocity, when substituted into Eq. (5) with $\beta$ replaced with zero. The unknowns may be readily established. They are:

$$
\begin{aligned}
K_{m} & =\frac{c_{m, 0}^{2}-c_{0}^{2}(\gamma-2)}{4 c_{m, 0}^{4}} \rho_{0}, \\
L_{m} & =\frac{c_{0}^{2}\left(c_{m, 0}^{2}(2 \gamma-1)-c_{0}^{2}(\gamma-2)\right)}{4 c_{m, 0}^{4}} \rho_{0}, \\
N_{m} & =\frac{\left(c_{m, 0}^{2}-c_{0}^{2}\right)\left(3 c_{m, 0}^{2}+c_{0}^{2}(\gamma-2)\right)}{4 c_{m, 0}^{2}} \rho_{0} .
\end{aligned}
$$

In the unmagnetised gas,

$$
K=-\frac{(\gamma-3) \rho_{0}}{4 c_{0}^{2}}, \quad L=\frac{\gamma+1}{4} \rho_{0}, \quad N=0
$$

These constants make the progressive Riemann's wave isentropic in the leading order (RUDEnKo, Soluyan, 1977); they also yield the parameter of nonlinearity

$$
\varepsilon=\frac{\gamma+1}{2}
$$

The parameter of nonlinearity, which is responsible for distortions of magnetoacoustic wave, see also paper by SHARMA et al. (1987), equals

$$
\varepsilon_{m}=\frac{3 c_{m, 0}^{2}+c_{0}^{2}(\gamma-2)}{2 c_{m, 0}^{2}} .
$$

The dynamic equation which accounts for nonlinear distortions and attenuation recalls the Burgers equation in newtonian fluids:

$$
\begin{aligned}
\frac{\partial v_{1}}{\partial t} & +c_{m, 0} \frac{\partial v_{1}}{\partial x}+\varepsilon_{m} v_{1} \frac{\partial v_{1}}{\partial x} \\
& -\frac{\left(c_{m, 0}^{2}-c_{0}^{2}\right) \beta}{2 c_{m, 0}^{2}} \frac{\partial^{2} v_{1}}{\partial x^{2}}=0
\end{aligned}
$$

Attenuation of magnetoacoustic wave depends in general on unperturbed magnetic pressure, $h_{0}$, by means of $c_{m, 0}$, and electrical conductivity and magnetic permeability, by means of $\beta$. This distinguishes Eq. (20) from the Burgers equation for the newtonian fluids, where attenuation depends on the summary damping due to shear, bulk viscosity, and thermal conduction. Solutions of Eq. (20) may be established by the well known methods: the Burgers equation readily transforms into the linear diffusion equation by the HopfCole transformation (Rudenko, Soluyan, 1977). The stationary solutions in the form of a shock wave which propagates with linear magnetoacoustic speed or with a different one, are also well known.

\subsection{High frequencies}

In this case, nonlinear corrections may be established by use of the same procedure which was described in the previous subsection. The nonlinear corrections in $\psi_{1}$ (when $B$ tends to zero),

$$
\begin{aligned}
\psi_{1} & =\left(\begin{array}{llll}
\rho_{1}^{\prime} & v_{1} & p_{1}^{\prime} & h_{1}^{\prime}
\end{array}\right)^{\mathrm{T}} \\
& =\left(\begin{array}{llll}
\frac{\rho_{0}}{c_{0}} & 1 & \rho_{0} c_{0} & 0
\end{array}\right)^{\mathrm{T}} v_{1},
\end{aligned}
$$

are determined by constants $K, L, N$ for unmagnetised gas. The parameter of nonlinearity also takes an unmagnitised value. The dynamic equation for velocity takes the form:

$$
\frac{\partial v_{1}}{\partial t}+c_{0} \frac{\partial v_{1}}{\partial x}+\varepsilon v_{1} \frac{\partial v_{1}}{\partial x}+\frac{B}{2}\left(c_{m, 0}^{2}-c_{0}^{2}\right) v_{1}=0 .
$$

It may be rearranged into a pure nonlinear equation in the new variables

$$
\begin{aligned}
\widetilde{v}_{1} & =\exp \left(B\left(c_{m, 0}^{2}-c_{0}^{2}\right) x /\left(2 c_{0}\right)\right) v_{1}, \\
X & =-\frac{2 c_{0}\left[\exp \left(-B\left(c_{m, 0}^{2}-c_{0}^{2}\right) x / 2 c_{0}\right)-1\right]}{B\left(c_{m, 0}^{2}-c_{0}^{2}\right)}, \\
\tau & =t-x / c_{0}
\end{aligned}
$$

with the leading-order result

$$
\frac{\partial \widetilde{v}_{1}}{\partial X}-\frac{\varepsilon}{c_{0}^{2}} \widetilde{v}_{1} \frac{\partial \widetilde{v}_{1}}{\partial \tau}=0 .
$$

Methods of solution of pure nonlinear equation Eq. (23) before and after forming of the discontinuities, are well known (Rudenko, Soluyan, 1977). It 
is interesting to note that Eq. (22) describes sound in many other cases of thermodynamic relaxation of internal degrees of freedom in gases (OsIPOV, UvAROV, 1992). Also, it appears in open systems with external heating/cooling and, with negative coefficient standing by $v_{1}$, describes sound in acoustically active media.

\section{Evolution of non-wave modes in the field of magnetoacoustic wave}

The projecting rows $P_{3}$ and $P_{4}$ eliminate terms specifying other modes in the linear part of Eq. (5) which describes dynamics of $\rho_{3}^{\prime}$ or $\rho_{4}^{\prime}$. They apply also to $\psi+\psi_{n l}$. Only terms belonging to the first progressive mode will be considered among all variety of nonlinear terms in $\psi_{n l}$. The terms which form the "magnetoacoustic forces" of the secondary modes originate from the nonlinear magnetoacoustic terms proportional to $\beta$ (or $B$ ) in the initial Eq. (5), and from the terms proportional to $\beta$ (or $B$ ) in the projectors.

\subsection{Low frequencies}

Application of $P_{3}$ yields an equation which governs an excess density in the entropy mode:

$\frac{\partial \rho_{3}^{\prime}}{\partial t}=\beta(\gamma-1) \rho_{0} \frac{\left(c_{m, 0}^{2}-c_{0}^{2}\right)}{2 c_{m, 0}^{4}}\left(\left(\frac{\partial v_{1}}{\partial x}\right)^{2}+v_{1} \frac{\partial^{2} v_{1}}{\partial x^{2}}\right)$.

Making use of $P_{4}$, we obtain the governing equation for an excess density corresponding to the fourth mode:

$$
\begin{aligned}
\frac{\partial \rho_{4}^{\prime}}{\partial t}-\frac{\beta c_{0}^{2}}{c_{m, 0}^{2}} \frac{\partial^{2} \rho_{4}^{\prime}}{\partial x^{2}}= & \beta \rho_{0} \frac{\left(c_{m, 0}^{2}-c_{0}^{2}\right)}{2 c_{m, 0}^{6}} \\
& \cdot\left(3 c_{0}^{2}(\gamma-2)-c_{m, 0}^{2} \gamma\right) \\
& \cdot\left(\left(\frac{\partial v_{1}}{\partial x}\right)^{2}+v_{1} \frac{\partial^{2} v_{1}}{\partial x^{2}}\right) .
\end{aligned}
$$

Equations (24), (25) describe nonlinear effects produced by periodic or aperiodic magnetoacoustic perturbations. They are not averaged over the sound period. On the other hand, $v_{1}$ should satisfy Eq. (20). Equation (24) may be integrated over time for approximately progressive with the speed $c_{m, 0}$ magnetoacoustic perturbation with the result:

$$
\rho_{3}^{\prime}=-\beta(\gamma-1) \rho_{0} \frac{\left(c_{m, 0}^{2}-c_{0}^{2}\right)}{2 c_{m, 0}^{5}} v_{1} \frac{\partial v_{1}}{\partial x} .
$$

The acoustic forces of heating and magnetic perturbation in the right-hand sides of Eqs. (24), (25) are neglible quantities in the case of nearly periodic sound since they equal zero on average. That is surprising in view of the fact that the dispersion relations, dynamic equation for sound, and links of $p_{1}^{\prime}, v_{1}$ and $\rho_{1}^{\prime}$ overlap in their form with those for newtonian flows (Eqs. (9), (10), (20)) (Perelomova, 2008). This is conditioned by the absence of nonlinear term proportional to $\beta$ in the dynamic equation for excess pressure analogous to newtonian attenuation. On the contrary, acoustic force of heating in a newtonian flow is proportional to $\left(\frac{\partial v_{1}}{\partial x}\right)^{2}$, which makes it almost constant on average for periodic perturbations. Hence, it depends on the sound intensity and total attenuation but does not depend on frequency of sound.

\subsection{High frequencies}

In the high-frequency limit, the dynamic equations which govern secondary modes, follow by making use of $P_{3}$ and $P_{4, h}$ :

$$
\frac{\partial \rho_{3}^{\prime}}{\partial t}=-B(\gamma-1) \rho_{0} \frac{\left(c_{m, 0}^{2}-c_{0}^{2}\right)}{2 c_{0}^{2}}\left(v_{1}^{2}+\frac{\partial v_{1}}{\partial x} \int v_{1} \mathrm{~d} x\right)
$$

and

$$
\frac{\partial^{2} h_{4}^{\prime}}{\partial x^{2}}-B \frac{\partial h_{4}^{\prime}}{\partial t}=O\left(B^{2}\right)
$$

The generation of magnetic pressure is a weak effect for both periodic and aperiodic magnetoacoustic perturbations, as well as for the magnetoacoustic heating. The acoustic force of heating is proportional to both small parameters, $B$ and $\left(c_{m, 0}^{2}-c_{0}^{2}\right) / c_{0}^{2}$. Equation (27) may be readily integrated with the leading-order result, which is almost zero on average for the periodic sound:

$$
\rho_{3}^{\prime}=B(\gamma-1) \rho_{0} \frac{\left(c_{m, 0}^{2}-c_{0}^{2}\right)}{2 c_{0}^{2} c_{m, 0}}\left(v_{1} \int v_{1} \mathrm{~d} x\right) .
$$

\section{Magnetoacoustic streaming in a two-dimensional quasi-planar flow}

We consider now velocity in the pane $(x, y)$, that is, $\mathbf{v}=\left(v_{x}(x, y, t), \quad v_{y}(x, y, t), 0\right)$ perpendicular to magnetic field $\mathbf{H}=\left(0,0, H_{z}(x, y, t)\right)$. We also consider a weakly diffracting magnetoacoustic beam which propagates, for definiteness, in the positive direction of axis $x$. A flow is characterised by a small parameter which accounts for diffraction and measures the ratio of characteristic scales of perturbations in the longitudinal and transversal directions (that is, the ratio of characteristic wavelenghts of sound and radius of a transducer), $\mu=k_{y} / k_{x}$, so that

$$
\sqrt{k_{x}^{2}+k_{y}^{2}} \approx k_{x}\left(1+\frac{k_{y}^{2}}{2 k_{x}^{2}}\right)
$$

etc. The analog of the Khokhlov-ZabolotskayaKuznetsov equation, which describes propagation of the weakly diffracting sound beam in a newtonian fluid (Rudenko, Soluyan, 1977), may be written on for 
the magnetoacoustic beam. In the case of the lowfrequency sound, it describes the longitudinal velocity in the magnetoacoustic beam:

$$
\begin{aligned}
\frac{\partial v_{1, x}}{\partial t} & +c_{m, 0} \frac{\partial v_{1, x}}{\partial x}+\varepsilon_{m} v_{1, x} \frac{\partial v_{1, x}}{\partial x} \\
& -\frac{\left(c_{m, 0}^{2}-c_{0}^{2}\right) \beta}{2 c_{m, 0}^{2}} \frac{\partial^{2} v_{1, x}}{\partial x^{2}} \\
& +c_{m, 0} \frac{\partial^{2}}{\partial y^{2}} \int v_{1, x} \mathrm{~d} x=0 .
\end{aligned}
$$

As for the vortex motion, it is determined by the fifth root of dispersion relation, $\omega_{5}=0$, which corresponds to the solenoidal flow, $\boldsymbol{\nabla} \cdot \mathbf{v}_{5}=0$. The solenoidal velocity attributable to the vortex flow may be decomposed from the total one by applying of the operator $P_{v o r}$ at the vector of the total velocity:

$$
\begin{aligned}
P_{v o r} \mathbf{v} & =\Delta^{-1}\left(\begin{array}{cc}
\frac{\partial^{2}}{\partial y^{2}} & -\frac{\partial^{2}}{\partial x \partial y} \\
-\frac{\partial^{2}}{\partial x \partial y} & \frac{\partial^{2}}{\partial x^{2}}
\end{array}\right)\left(\begin{array}{c}
\sum_{n=1}^{5} v_{n, x} \\
\sum_{n=1}^{5} v_{n, y}
\end{array}\right) \\
& =\left(\begin{array}{c}
v_{5, x} \\
v_{5, x}
\end{array}\right) .
\end{aligned}
$$

Applying $P_{v o r}$ at the two-dimensional momentum equation and considering the first magnetoacoustic wave as dominative, one arrives to the equation governing velocity of magnetoacoustic streaming:

$$
\begin{aligned}
\frac{\partial \mathbf{v}_{5}}{\partial t} & =-\frac{1}{\rho_{0}} P_{\text {vor }}\left(\rho_{1}^{\prime} \frac{\partial \mathbf{v}_{1}}{\partial t}\right) \\
& =\beta \frac{c_{m, 0}^{2}-c_{0}^{2}}{2 c_{m, 0} \rho_{0}^{2}} P_{\text {vor }} \rho_{1}^{\prime} \nabla \frac{\partial}{\partial x} \rho_{1}^{\prime} .
\end{aligned}
$$

The averaged form of equation which describes the longitudinal component of the streaming velocity in the case of the periodic magnetoacoustic wave may be expressed in terms of magnetoacoustic pressure:

$$
\overline{\frac{\partial v_{5, x}}{\partial t}}=F_{m, s}=\beta \frac{c_{m, 0}^{2}-c_{0}^{2}}{2 c_{m, 0}^{7} \rho_{0}^{2}} \overline{\left(\frac{\partial p_{1}^{\prime}}{\partial t}\right)^{2}} .
$$

$F_{m, s}$ overlaps in its form with the acoustic force of streaming in a newtonian fluid (PERELOMOVA, 2006; Makarov, Ochmann, 1996). The upper line denotes averaging over the sound period. The details of the evaluations in the case of a newtonian fluid may be found in (PERElomova, 2006; MAKArov, Ochmann, 1996; Perelomova, Wojda, 2010). The longitudinal velocity of streaming is directed according to the course of sound and enhances in time. The magnetoacoustic streaming induced by the high-frequency sound is fairly weak.

\section{Concluding remarks}

This study brings out some features of the generation of slow modes by the dominative sound in the magnetogasdynamic flow perpendicular to the magnetic field. Weakly nonlinear Eqs. (24), (25), (27), (28), (32) are the main results of the study. They are valid for periodic and aperiodic sound and describe evolution of the non-wave perturbations in the field of dominative sound independently on its spectrum (in the frames of the starting points). This makes the method different from the usual methods of seeking a solution as the series of harmonics and resolving of coupling equations for exactly satisfied resonance conditions with some desired accuracy (BRODIN et al., 2006; ZAVERSHINSKY, MoLEVICH, 2014).

The nonlinear effects of "low" and "high" frequency acoustic planar and quasi-planar waves are considered. These both cases formally coincide if

$$
c_{m, 0}^{2}-c_{0}^{2} \ll \omega \beta \ll \frac{c_{m, 0}^{4}}{c_{m, 0}^{2}-c_{0}^{2}} .
$$

This may be satisfied at extremely small magnetic strength. The leading-order dispersion relations in this case are simply

$$
\omega_{1}=c_{0} k, \quad \omega_{2}=-c_{0} k, \quad \omega_{3}=0, \quad \omega_{4}=i \beta k^{2} .
$$

This case differs slightly from the case of an unmagnitised flow. The weak dispersion followed by weak attenuation of magnetoacoustic perturbations is considered in this study as a reason for nonlinear distortion of sound and its coupling with the non-wave modes. This case is the most important in view of large spatial and temporal domains over which the magnetosound perturbations may be considered as dominative. Otherwise, magnetosound wave quickly decays.

The dispersion relations in the case of small magnetic strength, $c_{m, 0}^{2} / c_{0}^{2}-1 \ll 1$, and without any restrictions on magnetic permeability $\beta$, are

$$
\begin{aligned}
& \omega_{1}=c_{0} k+\frac{\left(c_{0}+i \beta k\right) k h_{0}}{c_{0}^{2} \rho_{0}+\beta^{2} k^{2} \rho_{0}}, \\
& \omega_{2}=-c_{0} k-\frac{\left(c_{0}-i \beta k\right) k h_{0}}{c_{0}^{2} \rho_{0}+\beta^{2} k^{2} \rho_{0}}, \\
& \omega_{3}=0, \\
& \omega_{4}=i \beta k^{2}-\frac{2 i \beta h_{0} k^{2}}{c_{0}^{2} \rho_{0}+\beta^{2} k^{2} \rho_{0}} .
\end{aligned}
$$

They readily determine modes inherent to this flow. In particular, the first magnetoacoustic mode takes the form 


$$
\begin{aligned}
& \psi_{1}=\left(\begin{array}{llll}
\rho_{1}^{\prime} & v_{1} & p_{1}^{\prime} & h_{1}^{\prime}
\end{array}\right)^{\mathrm{T}} \\
& =\left(\frac{\rho_{0}}{c_{0}}-\frac{2 h_{0}}{\beta c_{0}^{2}} \int_{-\infty}^{x} \exp \left(-c_{0}\left(x-x^{\prime}\right) / \beta\right) \mathrm{d} x^{\prime}\right. \\
& 1 \quad \rho_{0} c_{0}-\frac{h_{0}}{\beta} \int_{-\infty}^{x} \exp \left(-c_{0}\left(x-x^{\prime}\right) / \beta\right) \mathrm{d} x^{\prime} \\
& \left.\frac{2 h_{0}}{\beta} \int_{-\infty}^{x} \exp \left(-c_{0}\left(x-x^{\prime}\right) / \beta\right) \mathrm{d} x^{\prime}\right)^{\mathrm{T}} v_{1} .
\end{aligned}
$$

The equation which governs magnetosound velocity, takes the form

$$
\begin{aligned}
\frac{\partial v_{1}}{\partial t}+ & c_{m, 0} \frac{\partial v_{1}}{\partial x}+\varepsilon_{m} v_{1} \frac{\partial v_{1}}{\partial x}+\frac{h_{0}}{\beta \rho_{0}} \\
& \cdot \int_{-\infty}^{x} \frac{\partial v_{1}\left(x^{\prime}, t\right)}{\partial x^{\prime}} \exp \left(-c_{0}\left(x-x^{\prime}\right) / \beta\right) \mathrm{d} x^{\prime}=0 .
\end{aligned}
$$

The projecting rows into the fourth mode will take the form of double integrals with the limits which follow from the physical context of the flow, usually from $\infty$ till $x$. In view of that, further analysis of the nonlinear effects of sound is fairly difficult. Despite of this there are no obstacles to deriving coupling evolution equations making use of the method of projecting with an accurate account for dispersion caused by electrical conductivity.

The main conclusions about efficiency of nonlinear excitation of non-acoustic modes can be drawn without precise analysis. The dispersive properties of sound (established by Eqs. (34)) differ from that in the Maxwellian fluids with typical thermodynamic relaxation. In particular, the phase sound speed decreases with increasing wavenumber $k$ (or, equivalently, increasing frequency $\omega$ ), and attenuation is frequentlyindependent at large frequencies. The strongest attenuation of sound occurs at frequency $c_{0}^{2} / \beta$. I has been discovered that strong interaction between magnetic and hydrodynamic energies occurs at frequencies less than the frequency of maximum attenuation (ANDERSON, 1953). If it does not happen to low-frequency range, it is hardly expected to happen at other domain of frequencies. Magnetoacoustic heating and generation of the magnetic pressure in the Alfvén wave are expected to be insignificant when excited by periodic sound of any frequency or by the impulse sound. Acoustic streaming (excitation of mean stream in a gas) is similar to that in a newtonian fluid and is pronounced at low frequencies. The beam which is considered in Sec. 5, is actually transmitted by a rectangular transducer. That essentially simplifies the mathematical context as compared with a beam with circular crosssection: velocity of fluids in a beam with cylindrical symmetry cannot be perpendicular to one-dimensional magnetic field.

We do not consider in this study shear, bulk viscosity and thermal conductivity of a gas. These effects impose on those which are introduced by dispersion caused by electric conductivity of a plasma. In particular, they are of most importance in acoustic heating in a plasma. The conclusions concern also conducting liquids. Equations (24), (25), (26) are longer valid with $\gamma$ denoting the adiabatic coefficient of liquid. As for mercury, a magnetic flux density $H$ of 2.4 teslas yields absorption maximum about 0.5 nepers per wavelength at $5 \cdot 10^{5} \mathrm{~Hz}$. Water is much less conducting, and frequency of the strongest attenuation in it is very low, approximately $5 \mathrm{~Hz}$ (ANDERSON, 1953).

\section{References}

1. Anand R.K., Yadav H.C. (2014), On the structure of MHD shock waves in a viscous non-ideal gas, Theor. Comp. Fluid Dyn., 28, 369-376, doi: $10.1007 / \mathrm{s} 00162-014-0320-y$.

2. Anderson N.S. (1953), Longitudinal magnetohydrodynamic waves, JASA, 23, 3, 529-532, doi: 10.1121/1.1907074.

3. BAllai I. (2006), Nonlinear waves in solar plasmas a review, Journal of Physics: Conference Series, 44, 20, 20-29, doi: 10.1088/1742-6596/44/1/003.

4. Brodin G., Stenflo L., Shukla P.K. (2006), Nonlinear interactions between kinetic Alfven and ionsound waves, arXiv.physics/0604122v1 [physics.plasm-ph].

5. Fabian A.C., Reynolds C.S., Taylor G.B., DunN R.J.H. (2005), On viscosity, conduction and sound waves in the intracluster medium, Monthly Notices of the Royal Astronomical Society, 363 3, 891896, doi: 10.1111/j.1365-2966.2005.09484.x.

6. Geffen N. (1963), Magnetogasdynamic flows with shock waves, Phys. Fluids, 6, 4, 566-571, doi:10.1063/1.1706774.

7. Herlofson N. (1950), Magneto-hydrodynamic waves in a compressible fluid conductor, Nature, 165, 10201021, doi: 10.1038/1651020a0.

8. Krishna Prasad S., Banerjee D., Van DoorsSELAERE T. (2014), Frequency-dependent damping in propagating slow magnetoacoustic waves, Astrophys. Journ., 789, 118, 1-10, doi: 10.1088/0004-637X/789/2/118.

9. Korobeinikov V.P. (1976), Problems in the theory point explosion in gases, American Mathematical Society, Providence.

10. LeBLE S. (1991), Nonlinear waves in waveguides with stratification, Springer-Verlag, Berlin.

11. Makarov S., Ochmann M. (1996), Nonlinear and thermoviscous phenomena in acoustics I, Acta Acustica united with Acustica, 82, 4, 579-606. 
12. Osipov A.I., Uvarov A.V. (1992), Kinetic and gasdynamic processes in nonequilibrium molecular physics, Sov. Phys. Usp., 35, 11, 903-923.

13. Perelomova A. (2006), Development of linear projecting in studies of non-linear flow. Acoustic heating induced by non-periodic sound, Physics Letters A, 357, 42-47, doi: 10.1016/j.physleta.2006.04.014.

14. Perelomova A. (2008), Modelling of acoustic heating induced by different types of sound, Archives of Acoustics 33, 2, 151-160.

15. Perelomova A., Wojda P. (2010), Generation of the vorticity mode by sound in a relaxing Maxwell fluid, Acta Acustica united with Acustica, 96, 5, 807-813.

16. Petviashvili V.I., Pokhotelov O.A. (1992), Solitary waves in plasmas and in the atmosphere, Gordon and Breach, Berlin.

17. Ponomarev E.A. (1961), On the propogation of lowfrequency oscillations along the magnetic field in a viscous compressible plasma, Soviet Astronomy 5, 673676.

18. Rudenko O.V., Soluyan S.I. (1977), Theoretical foundations of nonlinear acoustics, Plenum, New York.

19. Sagdeev R.Z., Galeev A.A. (1969), Nonlinear plasma theory, Benjamin, New York.

20. Sharma V.D., Singh L.P., Ram R. (1987), The progressive wave approach analyzing the decay of a sawtooth profile in magnetogasdynamics, Phys. Fluds, 30, 5, 1572-1574, doi: 10.1063/1.866222.
21. Shukla P.K., Stenflo L. (1999), [in:] Nonlinear MHD Waves and Turbulence, Lecture Notes in Solar Phys., T. Passot, P.-L. Sulem [Eds.], 1-30, Springer, Berlin.

22. Shyam R., Sharma V.D., Sharma J. (1981), Growth and decay of weak waves in radiative magnetogasdynamics, AIAA Journal, 19, 9, 1246-1248, doi: $10.2514 / 3.60060$.

23. Singh L.P., Singh D.B., RAm S. (2011), Evolution of weak shock waves in perfectly conducting gases, Applied Mathematics, 2, 653-660, doi: 10.4236/am.2011.25086.

24. Singh L.P., Singh R., RAm S.D. (2012), Evolution and decay of acceleration waves in perfectly conducting inviscid radiative magnetogasdynamics, Astrophys. Space Sci., 342, 371-376, doi: 10.1007/s10509-012-1189-0.

25. Truesdell C. (1950), The effect of the compressibility of the Earth on its magnetic field, Phys. Rev., 78, 6, 823-823, doi: 10.1103/PhysRev.78.823.

26. Vincenti W.G., Baldwin JR B.S. (1962), Effect of thermal radiation on the propagation of plane acoustic waves, J. Fluid Mech., 12, 449-477.

27. Zavershinsky D.I., Molevich N.E. (2014), Alfven wave amplification as a result of nonlinear interaction with a magnetoacoustic wave in an acoustically active conducting medium, Technical Physics Letters, 40, 8, 701-703, doi: 10.1134/S1063785014080288. 